RAL-TR-96-020

March 1996

\title{
The spin-dependent two-loop splitting functions
}

\author{
W. Vogelsang \\ Rutherford Appleton Laboratory, \\ Chilton, DIDCOT, \\ Oxon OX11 0QX, England.
}

September 19, 2018

\begin{abstract}
We present a complete description of the calculation of the spin-dependent next-to-leading order splitting functions. The calculation is performed in the light-cone gauge. We give results for different prescriptions for the Dirac matrix $\gamma_{5}$ in $d=4-2 \epsilon$ dimensions and provide the link to the results in dimensional reduction.
\end{abstract}




\section{Introduction}

It has become standard to perform analyses of unpolarized parton distributions at nextto-leading order (NLO) accuracy of QCD. An indispensable ingredient for such analyses are the two-loop splitting functions (or anomalous dimensions) which appear in the NLO $Q^{2}$-evolution (GLAP [1.24) equations. Results (in the $\overline{\mathrm{MS}}$ scheme) for these have originally been obtained in [3], using the Operator Product Expansion (OPE) formalism, and in [幽] where the somewhat more efficient method developed in [6] was employed] which is based on the factorization properties of mass singularities and on the use of the axial gauge. In a recent publication [8] we have presented a detailed description of the calculation in the latter method which had never been fully documented. The main aim of our study was to elucidate the role played by the light-cone gauge and the technical aspects related to its use, and thus to underline the utility of this gauge for perturbative QCD calculations which had been questioned in the past 9,10$]$.

The method of [6, 迎] has recently also been applied to derive [11] the polarized two-loop splitting functions needed for the NLO $Q^{2}$-evolution of the spin-dependent parton densities of a longitudinally polarized hadron. Previous OPE results of [12] were confirmed. It is the purpose of this paper to provide a more detailed and complete description of our 'polarized' calculation [11]. Although many details of the calculation are the same as for the unpolarized case and can therefore be found in [8], there is a new ingredient in the polarized case which requires a closer inspection: The Dirac matrix $\gamma_{5}$ and the totally antisymmetric Levi-Civita tensor $\epsilon_{\mu \nu \rho \sigma}$ enter the calculation as projectors onto definite helicity states of the involved longitudinally polarized quarks and gluons. When dimensional regularization is used, a prescription for dealing with these (genuinely four-dimensional) quantities in $d=4-2 \epsilon$ dimensions has to be adopted which should be free of algebraic inconsistencies. Several such ' $\gamma_{5}$ schemes', considered to provide a consistent regularization, have been suggested in the literature 13, 14, 15]. Our calculation [11] was performed using the original definitions for $\gamma_{5}$ and $\epsilon_{\mu \nu \rho \sigma}$ of 13] (HVBM scheme) which is usually regarded as the most reliable prescription. Besides giving a more detailed account of our previous calculation we will also address the use of other $\gamma_{5}$ schemes such as 14, 15. Furthermore, we will provide the link to the results in dimensional reduction.

\section{The Calculation}

\section{$2.1 \quad$ Framework}

An outline of the method of [6, [4, 5] to calculate (NLO) splitting functions as well as a detailed description of the calculation in the unpolarized case have recently been given in [8]. We will therefore only focus on the new aspects arising in the polarized case, some of which have already been discussed in our previous paper [11.

As usual, all polarized quantities like cross sections etc. will be denoted by a ' $\Delta$ ', i.e.,

$$
\Delta M \equiv \frac{1}{4}(M(++)+M(--)-M(+-)-M(-+)),
$$

\footnotetext{
${ }^{1}$ Note that there is a discrepancy between the results of [3] and [5] which was resolved in 7 in favor of the calculation of [5].
} 
where ',+- ' stand for the helicities of the scattering incoming particles. The polarized parton distributions $\Delta f(f=q, g)$ are defined by

$$
\Delta f \equiv f^{+}-f^{-},
$$

$f^{+}\left(f^{-}\right)$denoting the density of parton-type $f$ with positive (negative) helicity in a nucleon with positive helicity. Omitting the ' $\Delta$ ' in Eqs. (1,.2) and taking the sum on the right-handsides, one recovers the analogous relations for the unpolarized cross sections and parton distributions.

The general strategy consists of first expanding the squared matrix element $\Delta M$ for polarized virtual photon-polarized quark (gluon) scattering into a ladder of two-particle irreducible (2PI) kernels [6] $C_{0}, K_{0}$,

$$
\Delta M=\Delta\left[C_{0}\left(1+K_{0}+K_{0}^{2}+K_{0}^{3}+\ldots\right)\right] \equiv \Delta\left[\frac{C_{0}}{1-K_{0}}\right] .
$$

We now choose the light-cone gauge by introducing a light-like vector $n\left(n^{2}=0\right)$ with $n \cdot A=0$. At the same time, $n$ is used to define the longitudinal direction:

$$
n \cdot p \equiv p n \neq 0, n \cdot t=p \cdot t=0
$$

where $p$ is the momentum of the incoming parton (taken to be massless), and $t$ is any vector in the transverse plane. In the light-cone gauge the $2 \mathrm{PI}$ kernels are finite before the integration over the sides of the ladder is performed. Collinear singularities therefore appear only when integrating over the lines connecting the rungs of the ladder [6]. This allows for projecting out the singularities by introducing the projector onto polarized physical states, $\Delta \mathcal{P}$. More precisely, $\Delta \mathcal{P}$ decouples the product, $\Delta(A B)$, of two successive $2 \mathrm{PI}$ kernels by projecting onto definite helicity states of the particle connecting the kernels and by setting this particle on-shell in $A$. Writing down explicitly the combinations of the helicities of the in- and outgoing partons and of the intermediate particle, one immediately obtains from Eq. (1) $\Delta(A \Delta \mathcal{P} B) \rightarrow \Delta A \Delta B$, i.e., the (decoupled) product of two polarized kernels. Thus $\Delta M$ can be written in the factorized form

$$
\Delta M=\Delta C \Delta \Gamma,
$$

where (introducing the modified kernel $\left.K=K_{0}\left(1-(1-\Delta \mathcal{P}) K_{0}\right)^{-1}\right)$

$$
\begin{aligned}
\Delta C & =\Delta C_{0} \frac{1}{1-(1-\Delta \mathcal{P}) K_{0}}, \\
\Delta \Gamma & =\frac{1}{1-\Delta \mathcal{P} K} \\
& \equiv 1+\Delta \mathcal{P} K_{0}+\Delta \mathcal{P} K_{0}(1-\Delta \mathcal{P}) K_{0}+\left(\Delta \mathcal{P} K_{0}\right)\left(\Delta \mathcal{P} K_{0}\right)+\ldots
\end{aligned}
$$

$\Delta C$ is interpreted as the (finite) short-distance cross section, whereas $\Delta \Gamma$ contains all (and only) mass singularities. Working in dimensional regularization $(d=4-2 \epsilon)$ in the $\overline{\mathrm{MS}}$ scheme one has explicitly:

$$
\Delta \Gamma_{i j}\left(x, \alpha_{s}, \frac{1}{\epsilon}\right)=Z_{j}\left[\delta(1-x) \delta_{i j}+x \operatorname{PP} \int \frac{d^{d} k}{(2 \pi)^{d}} \delta\left(x-\frac{n \cdot k}{p n}\right) \Delta U_{i} K \frac{1}{1-\Delta \mathcal{P} K} \Delta L_{j}\right],
$$


where 'PP' extracts the pole part of the expression on its right and $Z_{j}(j=q(g))$ is the residue of the pole of the full quark (gluon) propagator. $k$ is the momentum of the parton leaving the uppermost kernel in $\Delta \Gamma$; by definition of $n, x$ can be interpreted as the infinite-momentum frame (IMF) momentum fraction of $p$ carried by $k$. The spin-dependent projection operators onto physical states are given by

$$
\begin{aligned}
& \Delta U_{q}=-\frac{1}{4 n \cdot k} \gamma_{5} \not h, \quad \Delta L_{q}=-\not p \gamma_{5} \\
& \Delta U_{g}=i \epsilon^{\mu \nu \rho \sigma} \frac{n_{\rho} k_{\sigma}}{n \cdot k}, \quad \Delta L_{g}=i \epsilon^{\mu \nu \rho \sigma} \frac{p_{\rho} n_{\sigma}}{2 p n} .
\end{aligned}
$$

We see that the quantities $\gamma_{5}$ and $\epsilon^{\mu \nu \rho \sigma}$ appear, for which we will have to define a continuation to $d$ dimensions.

Finally, it can be shown [4] that the coefficient of the $1 / \epsilon$ pole of $\Delta \Gamma$ corresponds to the GLAP [1]:2] evolution kernels we are looking for:

$$
\Delta \Gamma_{q q}\left(x, \alpha_{s}, \epsilon\right)=\delta(1-x)-\frac{1}{\epsilon}\left(\frac{\alpha_{s}}{2 \pi} \Delta P_{q q}^{(0)}(x)+\frac{1}{2}\left(\frac{\alpha_{s}}{2 \pi}\right)^{2} \Delta P_{q q}^{(1)}(x)+\ldots\right)+O\left(\frac{1}{\epsilon^{2}}\right),
$$

and analogously for the flavor singlet case. Here we have adopted the perturbative expansion of the splitting functions,

$$
\Delta P_{i j}\left(x, \alpha_{s}\right)=\left(\frac{\alpha_{s}}{2 \pi}\right) \Delta P_{i j}^{(0)}(x)+\left(\frac{\alpha_{s}}{2 \pi}\right)^{2} \Delta P_{i j}^{(1)}(x)+\ldots .
$$

We conclude this section by collecting all ingredients for a NLO study of longitudinally polarized deep-inelastic scattering in terms of the spin-dependent structure function $g_{1}\left(x, Q^{2}\right)$. There are two different short-distance cross sections? $\Delta C_{q}$ and $\Delta C_{g}$, for scattering off incoming polarized quarks and gluons, respectively. They are to be calculated according to Eq. (6). Thus $g_{1}$ reads to next-to-leading order:

$$
\begin{aligned}
g_{1}\left(x, Q^{2}\right) & =\frac{1}{2} \sum_{i=1}^{n_{f}} e_{i}^{2}\left\{\Delta q_{i}\left(x, Q^{2}\right)+\Delta \bar{q}_{i}\left(x, Q^{2}\right)+\right. \\
& \left.+\frac{\alpha_{s}\left(Q^{2}\right)}{2 \pi}\left[\Delta C_{q} \otimes\left(\Delta q_{i}+\Delta \bar{q}_{i}\right)+\frac{1}{n_{f}} \Delta C_{g} \otimes \Delta g\right]\left(x, Q^{2}\right)\right\},
\end{aligned}
$$

where $n_{f}$ is the number of flavors and $\otimes$ denotes the convolution

$$
f \otimes g \equiv \int_{0}^{1} d y d z f(y) g(z) \delta(x-y z) .
$$

Defining the sum and the difference of polarized quark and antiquark distributions as

$$
\Delta q_{i}^{ \pm}=\Delta q_{i} \pm \Delta \bar{q}_{i}
$$

one finds the following evolution equations for the non-singlets $\Delta q_{i}^{-}$and $\Delta q_{i}^{+}-\Delta q_{j}^{+}$(see, e.g., [8]):

$$
\begin{aligned}
\frac{d}{d \ln Q^{2}}\left(\Delta q_{i}^{+}-\Delta q_{j}^{+}\right)\left(x, Q^{2}\right) & =\Delta P_{q q}^{+}\left(x, \alpha_{s}\left(Q^{2}\right)\right) \otimes\left(\Delta q_{i}^{+}-\Delta q_{j}^{+}\right)\left(x, Q^{2}\right) \\
\frac{d}{d \ln Q^{2}} \Delta q_{i}^{-}\left(x, Q^{2}\right) & =\Delta P_{q q}^{-}\left(x, \alpha_{s}\left(Q^{2}\right)\right) \otimes \Delta q_{i}^{-}\left(x, Q^{2}\right)
\end{aligned}
$$

\footnotetext{
${ }^{2}$ We do not distinguish between a quark non-singlet and a quark singlet short-distance cross section here.
} 
where

$$
\Delta P_{q q}^{ \pm} \equiv \Delta P_{q q}^{V} \pm \Delta P_{q \bar{q}}^{V},
$$

with $\Delta P_{q \bar{q}}^{V}$ starting to be different from zero beyond the leading order. Introducing the polarized quark singlet $\Delta \Sigma \equiv \sum_{i}\left(\Delta q_{i}+\Delta \bar{q}_{i}\right)$ one has in the singlet sector:

$\frac{d}{d \ln Q^{2}}\left(\begin{array}{c}\Delta \Sigma\left(x, Q^{2}\right) \\ \Delta g\left(x, Q^{2}\right)\end{array}\right)=\left(\begin{array}{cc}\Delta P_{q q}\left(x, \alpha_{s}\left(Q^{2}\right)\right) & \Delta P_{q g}\left(x, \alpha_{s}\left(Q^{2}\right)\right) \\ \Delta P_{g q}\left(x, \alpha_{s}\left(Q^{2}\right)\right) & \Delta P_{g g}\left(x, \alpha_{s}\left(Q^{2}\right)\right)\end{array}\right) \otimes\left(\begin{array}{c}\Delta \Sigma\left(x, Q^{2}\right) \\ \Delta g\left(x, Q^{2}\right)\end{array}\right)$.

The $q q$ entry in the singlet matrix of splitting functions is given by

$$
\Delta P_{q q}=\Delta P_{q q}^{+}+\Delta P_{q q}^{S} .
$$

So, at NLO, we will have to derive the splitting functions $\Delta P_{q q}^{ \pm,(1)}, \Delta P_{q q}^{S,(1)}$, and those involving gluons.

\section{$2.2 \quad$ NLO graphs}

According to Eqs. (7] 8 ), $\Delta \Gamma$ is given to NLO by

$$
\Delta \Gamma=Z_{j}\left(1+\Delta \mathcal{P} K_{0}+\Delta \mathcal{P}\left(K_{0}^{2}\right)-\Delta \mathcal{P}\left(K_{0} \Delta \mathcal{P} K_{0}\right)\right) .
$$

The basic topologies of all 2PI diagrams which occur in NLO are shown in Fig. 1, where the notation of the topologies is as in [8]. Explicit examples of graphs contributing to the various splitting functions can be found in [4, 11, 87. Topologies (hi) correspond to the terms $\Delta \mathcal{P}\left(K_{0}^{2}\right)-\Delta \mathcal{P}\left(K_{0} \Delta \mathcal{P} K_{0}\right)$ in Eq. (20), all other topologies belong to $\Delta \mathcal{P} K_{0}$. As is obvious, topologies (cd) and (fg) possess a real and a virtual cut. Fig. 1 does not display the genuine two-loop graphs which determine $Z_{j}$ and thus the endpoint $(\delta(1-x))$ contributions to the diagonal splitting functions.

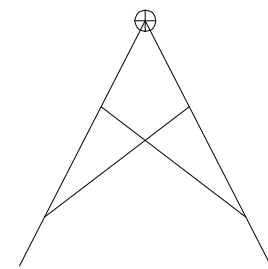

(b)

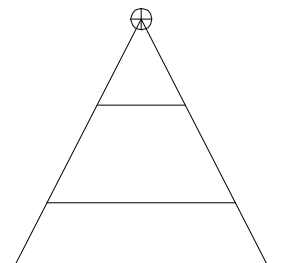

(hi)

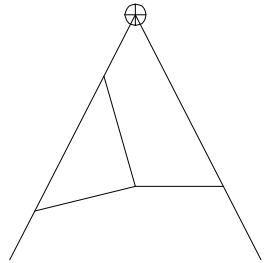

(cd)

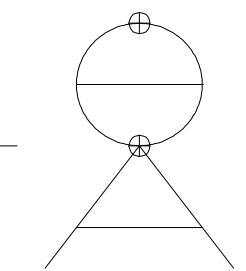

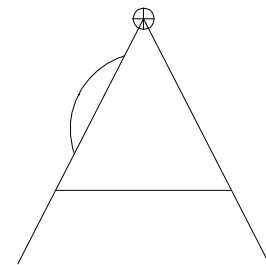

(e)

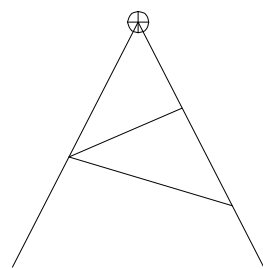

$(j k)$

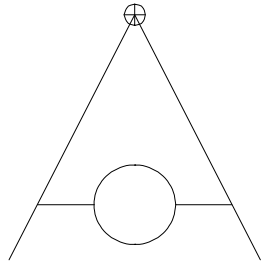

(fg)

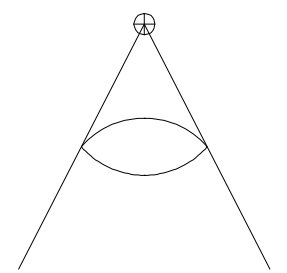

Figure 1: Basic topologies of the NLO diagrams

\footnotetext{
${ }^{3}$ As compared to 8 we include a factor $2 n_{f}$ in the definition of $\Delta P_{q q}^{S}$.
} 


\subsection{Use of the light-cone gauge}

As noted in the previous section, the light-cone gauge plays a crucial role in the calculation. In this gauge the gluon propagator takes the form

$$
\mathcal{D}^{\mu \nu}(l)=\frac{i}{l^{2}}\left(-g^{\mu \nu}+\frac{n^{\mu} l^{\nu}+n^{\nu} l^{\mu}}{n \cdot l}\right),
$$

where $l$ is the gluon's momentum. As is well-known, the light-cone gauge propagator $1 /(n \cdot l)$ can give rise to additional divergencies in loop and phase space integrals. We follow [4, 5, 8] to use the principal value $(\mathrm{PV})$ prescription to regulate such poles:

$$
\frac{1}{n \cdot l} \rightarrow \frac{1}{2}\left(\frac{1}{n \cdot l+i \delta(p n)}+\frac{1}{n \cdot l-i \delta(p n)}\right)=\frac{n \cdot l}{(n \cdot l)^{2}+\delta^{2}(p n)^{2}} .
$$

All singularities related to the gauge propagator can then be cast into the basic integrals 叫, 阳

$$
I_{i}=\int_{0}^{1} d u \frac{u \ln ^{i} u}{u^{2}+\delta^{2}} \quad(i=0,1) .
$$

The PV prescription leads to the feature that the renormalization constants depend on $I_{0}$ and the longitudinal momentum fractions $x$, 4,8$]$. The complete set of the renormalization constants, as well as most other technical ingredients we need for our calculation, like phase space integrals and scalar virtual integrals with and without a gauge propagator, have been worked out and collected in [8].

\subsection{Treatment of $\gamma_{5}$ and $\epsilon_{\mu \nu \rho \sigma}$}

As seen from Eq. (9), the crucial difference with respect to the unpolarized calculation is the presence of $\gamma_{5}$ and $\epsilon_{\mu \nu \rho \sigma}$ which act as projectors. Definitions for these quantities in $d=4-2 \epsilon$ dimensions (or at least rules for handling them) have to be given.

It is well known that a 'naive' fully anticommuting $d$-dimensional $\gamma_{5}$ can easily lead to algebraic inconsistencies when located in a trace with six or more other Dirac matrices [16], and thus results obtained this way cannot really be trusted. The problem can be avoided in essentially two ways:

- One can define $\gamma_{5}$ in $d$ dimensions by maintaining its four-dimensional definition

$$
\gamma_{5} \equiv \frac{i}{4 !} \epsilon^{\mu \nu \rho \sigma} \gamma_{\mu} \gamma_{\nu} \gamma_{\rho} \gamma_{\sigma}
$$

where the $\epsilon$-tensor is regarded as a genuinely four-dimensional object, i.e., its components vanish in all unphysical dimensions. This definition of $\gamma_{5}$ is the original one of [13] (HVBM scheme). Splitting the $d$-dimensional metric tensor into its four- and $(d-4)$-dimensional components,

$$
g_{\mu \nu}=\hat{\hat{g}}_{\mu \nu}+\hat{g}_{\mu \nu} \quad\left(\text { where } \hat{\hat{g}}_{\mu}^{\mu}=4, \hat{g}_{\mu}^{\mu}=d-4\right),
$$

and defining $\hat{\gamma}_{\mu} \equiv \hat{g}_{\mu \nu} \gamma^{\nu}$ etc., one finds from (24) that

$$
\begin{aligned}
\left\{\gamma^{\mu}, \gamma_{5}\right\} & =0 \text { for } \mu=0,1,2,3 \\
{\left[\gamma^{\mu}, \gamma_{5}\right] } & =0 \text { otherwise } .
\end{aligned}
$$


- One can maintain the full anticommutativity of $\gamma_{5}$ with all other Dirac matrices but abandon cyclicity of the trace operation [14]. In this case a fixed 'reading point' has to be defined from which all Dirac traces corresponding to a given process have to be started. As in the HVBM scheme, the $\epsilon$-tensor is defined as an entirely fourdimensional object [14].

Our main calculation ${ }^{4}$ is done in the HVBM scheme since its consistency is well-established. We will also discuss the results obtained in the scheme of [14] which was used in the OPE calculation of the $\Delta P_{i j}^{(1)}$ of 12]. Due to the fact that the Levi-Civita tensor is assumed to be four-dimensional (and because of (26) in the HVBM scheme), the matrix element of a graph will for both prescriptions not only depend on the usual ' $d$-dimensional' scalar products of two momenta, like $l \cdot k \equiv g_{\mu \nu} l^{\mu} k^{\nu}$ etc., but also on ' $(d-4)$-dimensional' ones, denoted by $\hat{l} \cdot \hat{k} \equiv \hat{g}_{\mu \nu} l^{\mu} k^{\nu}, \hat{k}^{2}$ etc. Special care has to be taken to take into account such $(d-4)$-dimensional terms in loop and phase space integrals. Details are given in the appendices.

We mention that in 15, 18 another prescription was suggested to handle traces with one $\gamma_{5}$. One eliminates $\gamma_{5}$ via the relation

$$
\gamma_{\mu} \gamma_{5}=\frac{i}{3 !} \epsilon_{\mu \nu \rho \sigma} \gamma^{\nu} \gamma^{\rho} \gamma^{\sigma}
$$

The remaining trace is then perfectly well-defined. One always ends up with the product of two Levi-Civita tensors which can be written as a determinant of a matrix, the elements of which are metric tensors. Following [15,18], one then performs the contractions of these metric tensors in $d$ dimensions?. The result should then coincide with that in the HVBM scheme. The scheme is attractive because it avoids any $(d-4)$-dimensional scalar products, but it leads to longer trace operations because of the substitution (27). We will briefly return to this prescription when presenting our results.

We finally note that a variant of dimensional regularization, dimensional reduction [19], has been widely discussed and used in the past years, the main reason being its applicability to supersymmetry. The scheme essentially consists of performing the Dirac-algebra in four dimensions, which makes the treatment of $\gamma_{5}$ straightforward, and of continuing only momenta to $d(d<4)$ dimensions. In order to match the ultraviolet (UV) sectors of dimensional regularization and dimensional reduction, specific counterterms have to be introduced [20,21] in the latter. Once this is done, there is a straightforward and universal way to deal with differences arising from mass singularities [21,22, 23]. We will exploit the results of [21,22,23] in order to translate our results to the form they take in dimensional reduction.

\section{Results}

The full one-loop results are included for completeness [1,24]:

$$
\Delta P_{q q}^{(0)}(x)=C_{F}\left\{\frac{2}{[1-x]_{+}}-1-x+\frac{3}{2} \delta(1-x)\right\}
$$

\footnotetext{
${ }^{4}$ We use the program TRACER of [17] for calculating the Dirac traces and performing contractions.

${ }^{5}$ Here one has to multiply the gluonic projection operator $\Delta L_{g}$ in (9) by a normalization factor $2 /((d-2)(d-3))$.
} 


$$
\begin{aligned}
& \Delta P_{q g}^{(0)}(x)=2 T_{f}\{2 x-1\} \\
& \Delta P_{g q}^{(0)}(x)=C_{F}\{2-x\} \\
& \Delta P_{g g}^{(0)}(x)=2 N_{C}\left\{\frac{1}{[1-x]_{+}}-2 x+1\right\}+\frac{\beta_{0}}{2} \delta(1-x),
\end{aligned}
$$

where

$$
C_{F}=\frac{4}{3}, N_{C}=3, T_{f}=T_{R} n_{f}=\frac{1}{2} n_{f}, \beta_{0}=\frac{11}{3} N_{C}-\frac{4}{3} T_{f}
$$

\subsection{Results in the HVBM scheme}

The graph-by-graph results (for those splitting functions that involve more than just one topology) in the HVBM scheme are given in columns (b)-(jk) of Tables 1-4. For the nonsinglet case, Table 1, we only present the differences between our graph-by-graph results for the polarized $\Delta P_{q q}^{V,(1)}$ and the corresponding contributions to the unpolarized $P_{q q}^{V,(1)}$ as listed in Table 1 of $\left[4\right.$. The reason for this is that for an anticommuting $\gamma_{5}$ the $q q$ diagrams would trivially yield $\Delta P_{q q}^{V,(1)}=P_{q q}^{V,(1)}$ because of the fact that there are always two $\gamma_{5}$ in the same trace which could be anticommuted towards each other and eliminated using $\gamma_{5}^{2}=1$. However, as discussed in section $2.4, \gamma_{5}$ does not fully anticommute in the HVBM scheme. So it is interesting to see what happens to the difference $\Delta P_{q q}^{V,(1)}-P_{q q}^{V,(1)}$ when this prescription is used. To examine this question further we have also distinguished in Table 1 the contributions coming from the $(d-4)$-dimensional scalar products, like $\hat{k}^{2}$ etc. (see section 2.4 and the appendices), from all other contributions?

Let us concentrate on the columns 'Sum' in each table, which give the sums of the graph-by-graph results (b)-(jk). The first thing to observe is that all contributions from the integrals $I_{0}, I_{1}$, arising from the light-cone gauge propagator, cancel in 'Sum', as they must. We furthermore see from Table 1 that $\Delta P_{q q}^{V,(1)}-P_{q q}^{V,(1)}$ is indeed non-vanishing in the terms multiplied by $C_{F} N_{C}$ and $C_{F} T_{f}$. To study the implications of this let us first note that the endpoint contributions $(\sim \delta(1-x))$ to the diagonal splitting functions are necessarily the same as in the unpolarized case (where they were derived in [25]8]), since they are determined by $Z_{j}$ in Eq. (8). We can then express the results corresponding to the columns 'Sum' in terms of the unpolarized NLO non-singlet splitting functions $P_{q q}^{ \pm,(1)}$ of [i] and the recent polarized OPE results $\Delta \tilde{P}_{i j}^{(1)}$ of 12 ]:

$$
\begin{aligned}
\Delta P_{q q}^{ \pm,(1)}(x) & =P_{q q}^{\mp,(1)}(x)-2 \beta_{0} C_{F}(1-x) \\
\Delta P_{q q}^{S,(1)}(x) & =\Delta \tilde{P}_{q q}^{S,(1)}(x) \\
\Delta P_{q g}^{(1)}(x) & =\Delta \tilde{P}_{q g}^{(1)}(x)+4 C_{F}(1-x) \otimes \Delta P_{q g}^{(0)}(x) \\
\Delta P_{g q}^{(1)}(x) & =\Delta \tilde{P}_{g q}^{(1)}(x)-4 C_{F}(1-x) \otimes \Delta P_{g q}^{(0)}(x) \\
\Delta P_{g g}^{(1)}(x) & =\Delta \tilde{P}_{g g}^{(1)}(x) \\
\Delta C_{q}(x) & =\Delta \tilde{C}_{q}(x)-4 C_{F}(1-x) \\
\Delta C_{g}(x) & =\Delta \tilde{C}_{g}(x)
\end{aligned}
$$

\footnotetext{
${ }^{6}$ In all other tables the contributions from the $(d-4)$-dimensional scalar products are already included.

${ }^{7}$ In our normalization given by Eq. 11, 12] the results for the NLO splitting functions of [12] have to be divided by 8 and those for the NLO short-distance cross sections $\Delta C_{q}, \Delta C_{g}$ by $\alpha_{s} / 2 \pi$.
} 
where we have also included the results for the short-distance cross sections $\Delta C_{q}, \Delta C_{g}$ which are to be calculated according to Eq. (6). Obviously, the term $-2 \beta_{0} C_{F}(1-x)$ in (33) is entirely due to the fact the HVBM $\gamma_{5}$ does not fully anticommute. As was already discussed in [27,28] and indicated in Eq. (33), the ' + ' and '-' combinations of the NS splitting functions as defined in (17) interchange their role when going from the unpolarized to the polarized case, equivalent to $\Delta P_{q \bar{q}}^{V,(1)}=-P_{q \bar{q}}^{V,(1)}$. The latter relation is a consequence of the projection operator for antiquarks [ $4,+\gamma_{5}(-\not h) / 4 n \cdot k$, in this case. Again it is trivial for a fully anticommuting $\gamma_{5}$, but it turns out that it is also respected by the HVBM $\gamma_{5}$. Eqs. 15, 17, 33) therefore imply that the combination $\Delta P_{q q}^{+,(1)}=P_{q q}^{-,(1)}-2 \beta_{0} C_{F}(1-x)$ would govern the $Q^{2}$-evolution of, e.g., the polarized NS quark combination

$$
\Delta A_{3}\left(x, Q^{2}\right)=\left(\Delta u^{+}-\Delta d^{+}\right)\left(x, Q^{2}\right) .
$$

Since the first moment (i.e., the $x$-integral) of the latter corresponds to the nucleon matrix element of the NS axial vector current $\bar{q} \gamma^{\mu} \gamma_{5} \lambda_{3} q$ which is conserved, it has to be $Q^{2}$ independent [29]. Keeping in mind that the integral of the unpolarized $P_{q q}^{-,(1)}$ vanishes already due to fermion number conservation [幽, it becomes obvious that the additional term $-2 \beta_{0} C_{F}(1-x)$ in (33) spoils the $Q^{2}$-independence of the first moment of $\Delta A_{3}\left(x, Q^{2}\right)$. It is therefore necessary to perform a factorization scheme transformation to the results in (33 39) in order to remove this additional term. Such a scheme transformation is always allowed since neither the $\Delta P_{i j}^{(1)}$ nor the $\Delta C_{i}$ are physical quantities. Thus one can shift terms between them in a well-defined way without changing a physical quantity like $g_{1}$, hereby just redefining the polarized NLO parton distributions. Even though it is a priori only the term $-2 \beta_{0} C_{F}(1-x)$ in (33) we want to remove, the transformation will also affect the singlet sector since, according to Eq. (19),$\Delta P_{q q}^{+,(1)}$ also occurs in the singlet evolution matrix. The scheme transformation reads in general (see, e.g., [30.12]):

$$
\begin{aligned}
\Delta P_{q q}^{ \pm,(1)} & \longrightarrow \Delta P_{q q}^{ \pm,(1)}-2 \beta_{0} \Delta z_{q q}, \\
\Delta P_{q q}^{(1)} & \longrightarrow \Delta P_{q q}^{(1)}-2 \beta_{0} \Delta z_{q q}+4 \Delta z_{q g} \otimes \Delta P_{g q}^{(0)}-4 \Delta z_{g q} \otimes \Delta P_{q g}^{(0)}, \\
\Delta P_{q g}^{(1)} & \longrightarrow \Delta P_{q g}^{(1)}-2 \beta_{0} \Delta z_{q g}+4 \Delta z_{q g} \otimes\left(\Delta P_{g g}^{(0)}-\Delta P_{q q}^{(0)}\right)+4 \Delta P_{q g}^{(0)} \otimes\left(\Delta z_{q q}-\Delta z_{g g}\right), \\
\Delta P_{g q}^{(1)} & \longrightarrow \Delta P_{g q}^{(1)}-2 \beta_{0} \Delta z_{g q}+4 \Delta z_{g q} \otimes\left(\Delta P_{q q}^{(0)}-\Delta P_{g g}^{(0)}\right)+4 \Delta P_{g q}^{(0)} \otimes\left(\Delta z_{g g}-\Delta z_{q q}\right), \\
\Delta P_{g g}^{(1)} & \longrightarrow \Delta P_{g g}^{(1)}-2 \beta_{0} \Delta z_{g g}-4 \Delta z_{q g} \otimes \Delta P_{g q}^{(0)}+4 \Delta z_{g q} \otimes \Delta P_{q g}^{(0)}, \\
\Delta C_{q} & \longrightarrow \Delta C_{q}-4 \Delta z_{q q}, \\
\Delta C_{g} & \longrightarrow \Delta C_{g}-4 \Delta z_{q g},
\end{aligned}
$$

where the $\Delta z_{i j}$ generate the transformation. For the case at hand we only need a nonvanishing $\Delta z_{q q}$,

$$
\Delta z_{q q}(x)=-C_{F}(1-x) .
$$

The changes to our result caused by inserting $\Delta z_{q q}$ into (40) are given in the columns " $\gamma_{5}$ " in Tables 1-3, to be added to columns 'Sum' to obtain the final answer. As one can see from Table 1, the difference $\Delta P_{q q}^{V,(1)}-P_{q q}^{V,(1)}$ finally becomes zero, which is a result of cancellations between terms from $(d-4)$-dimensional scalar products and other terms. Furthermore, it turns out that the transformation (40,41) not only removes the term $-2 \beta_{0} C_{F}(1-x)$ from Eq. (33), but eliminates all extra $(1-x)$-terms on the r.h.s. of (33 39), leaving $\Delta P_{q q}^{S,(1)}$, 
$\Delta P_{g g}^{(1)}$ and $\Delta C_{g}$ unchanged. Thus our final results are in complete agreement with those of [12]. We finally note that the presence of the $(1-x)$-terms in our original HVBM scheme result (33 39) can be traced back to the fact that in this scheme the polarized $L O$ splitting function in $d=4-2 \epsilon$ dimensions, $\Delta P_{q q}^{(0), d=4-2 \epsilon}$, is no longer equal to its unpolarized counterpart, i.e., violates helicity conservation:

$$
\Delta P_{q q}^{(0), d=4-2 \epsilon}(x)-P_{q q}^{(0), d=4-2 \epsilon}(x)=4 \epsilon(1-x) .
$$

The additional term $-4 C_{F}(1-x)$ in the HVBM-scheme result for $\Delta C_{q}$ in (38) was already identified in 31,28]. After its removal by the transformation (40,41), the integral over $\Delta C_{q}$ takes the value $-3 C_{F} / 2$, giving rise to the correct NLO correction $\left(1-\alpha_{s} / \pi\right)$ to, e.g., the Bjørken sum rule.

Our complete final results can now be collected from columns 'final' (or 'Sum' if there has been no change due to (40,41)) in Tables 1-4. To write them down we introduce

$$
\begin{aligned}
\delta p_{q g}(x) & \equiv 2 x-1 \\
\delta p_{g q}(x) & \equiv 2-x \\
\delta p_{g g}(x) & \equiv \frac{1}{[1-x]_{+}}-2 x+1 .
\end{aligned}
$$

We then have

$$
\begin{aligned}
\Delta P_{q q}^{ \pm,(1)}= & P_{q q}^{\mp,(1)}, \\
\Delta P_{q q}^{S,(1)}(x)= & 2 C_{F} T_{f}\left[(1-x)-(1-3 x) \ln x-(1+x) \ln ^{2} x\right] \\
\Delta P_{q g}^{(1)}(x)= & C_{F} T_{f}[-22+27 x-9 \ln x+8(1-x) \ln (1-x) \\
& \left.+\delta p_{q g}(x)\left(2 \ln ^{2}(1-x)-4 \ln (1-x) \ln x+\ln ^{2} x-\frac{2}{3} \pi^{2}\right)\right] \\
& +N_{C} T_{f}[2(12-11 x)-8(1-x) \ln (1-x)+2(1+8 x) \ln x \\
& \left.-2\left(\ln ^{2}(1-x)-\frac{\pi^{2}}{6}\right) \delta p_{q g}(x)-\left(2 S_{2}(x)-3 \ln ^{2} x\right) \delta p_{q g}(-x)\right] \\
\Delta P_{g q}^{(1)}(x)= & C_{F} T_{f}\left[-\frac{4}{9}(x+4)-\frac{4}{3} \delta p_{g q}(x) \ln (1-x)\right] \\
& +C_{F}^{2}\left[-\frac{1}{2}-\frac{1}{2}(4-x) \ln x-\delta p_{g q}(-x) \ln (1-x)\right. \\
& \left.+\left(-4-\ln ^{2}(1-x)+\frac{1}{2} \ln ^{2} x\right) \delta p_{g q}(x)\right] \\
& +C_{F} N_{C}\left[(4-13 x) \ln x+\frac{1}{3}(10+x) \ln (1-x)+\frac{1}{9}(41+35 x)\right. \\
& +\frac{1}{2}\left(-2 S_{2}(x)+3 \ln { }^{2} x\right) \delta p_{g q}(-x)
\end{aligned}
$$




$$
\begin{aligned}
& \left.+\left(\ln ^{2}(1-x)-2 \ln (1-x) \ln x-\frac{\pi^{2}}{6}\right) \delta p_{g q}(x)\right] \\
\Delta P_{g g}^{(1)}(x)= & -N_{C} T_{f}\left[4(1-x)+\frac{4}{3}(1+x) \ln x+\frac{20}{9} \delta p_{g g}(x)+\frac{4}{3} \delta(1-x)\right] \\
& -C_{F} T_{f}\left[10(1-x)+2(5-x) \ln x+2(1+x) \ln ^{2} x+\delta(1-x)\right] \\
+ & N_{C}^{2}\left[\frac{1}{3}(29-67 x) \ln x-\frac{19}{2}(1-x)+4(1+x) \ln ^{2} x-2 S_{2}(x) \delta p_{g g}(-x)\right. \\
& \left.+\left(\frac{67}{9}-4 \ln (1-x) \ln x+\ln ^{2} x-\frac{\pi^{2}}{3}\right) \delta p_{g g}(x)+\left(3 \zeta(3)+\frac{8}{3}\right) \delta(1-x)\right], \\
\Delta C_{q}(x)= & C_{F}\left[\left(1+x^{2}\right)\left[\frac{\ln (1-x)}{1-x}\right]_{+}-\frac{3}{2} \frac{1}{[1-x]_{+}}-\frac{1+x^{2}}{1-x} \ln x+\right. \\
& \left.\quad+2+x-\left(\frac{9}{2}+\frac{\pi^{2}}{3}\right) \delta(1-x)\right], \\
\Delta C_{g}(x)= & 2 T_{f}\left[(2 x-1)\left(\ln \frac{1-x}{x}-1\right)+2(1-x)\right]
\end{aligned}
$$

where the unpolarized NS pieces $P_{q q}^{\mp,(1)}$ can be found in 4,8 , and

$$
S_{2}(x)=\int_{\frac{x}{1+x}}^{\frac{1}{1+x}} \frac{d z}{z} \ln \left(\frac{1-z}{z}\right) .
$$

For relating the above results to those of [12] the relation

$$
S_{2}(x)=-2 \operatorname{Li}_{2}(-x)-2 \ln x \ln (1+x)+\frac{1}{2} \ln ^{2} x-\frac{\pi^{2}}{6}
$$

is needed, where $\operatorname{Li}_{2}(x)$ is the Dilogarithm [26]. As explained above, the $\delta(1-x)$-endpoint contributions could be taken from the unpolarized case $25 ., 8] ; \zeta(3) \approx 1.202057$. The +prescription in (43,49) is defined in the usual way,

$$
\int_{0}^{1} d z f(z)[g(z)]_{+} \equiv \int_{0}^{1} d z(f(z)-f(1)) g(z) .
$$

It is obviously only needed if the function multiplying it is non-vanishing at $x=1$. 50):

For completeness we finally list the first moments ${ }^{8}$ ( $x$-integrals) of the results in Eqs. (45.

$$
\int_{0}^{1} \Delta P_{q q}^{S,(1)}(x) d x=-3 C_{F} T_{f}, \quad \int_{0}^{1} \Delta P_{g q}^{(1)}(x) d x=-\frac{9}{4} C_{F}^{2}+\frac{71}{12} N_{C} C_{F}-\frac{1}{3} C_{F} T_{f},
$$

\footnotetext{
${ }^{8}$ Compact expressions for all Mellin-moments of the NLO splitting functions, $\int_{0}^{1} d x x^{n-1} \Delta P_{i j}^{(1)}(x)$, and their analytic continuations, can be found in 27.
} 


$$
\begin{aligned}
& \int_{0}^{1} \Delta P_{q g}^{(1)}(x) d x=0, \quad \int_{0}^{1} \Delta P_{g g}^{(1)}(x) d x=\frac{17}{6} N_{C}^{2}-C_{F} T_{f}-\frac{5}{3} N_{C} T_{f} \equiv \frac{\beta_{1}}{4}, \\
& \int_{0}^{1} \Delta C_{q}(x) d x=-\frac{3}{2} C_{F}, \quad \int_{0}^{1} \Delta C_{g}(x) d x=0 .
\end{aligned}
$$

\subsection{Results for other $\gamma_{5}$ prescriptions}

We now discuss the results we obtain when using the prescription of [14] with an anticommuting $\gamma_{5}$ and a non-cyclic trace. As mentioned earlier, the property $\left\{\gamma^{\mu}, \gamma_{5}\right\}=0$ will automatically yield $\Delta P_{q q}^{ \pm,(1)}=P_{q q}^{\mp,(1)}$ instead of (33). Thus it immediately follows that either all other $(1-x)$-terms in $(33-39)$ are absent as well, or that the full result will be genuinely different from the one in Eqs. (44 50), i.e., not transformable into 44 50) by a factorization scheme transformation (40). To study this question, we have to calculate the other NLO splitting functions and the short-distance cross sections. Since the prescription for the Levi-Civita tensor is the same as in the HVBM scheme, it is obvious that $\Delta P_{g g}^{(1)}$ and $\Delta C_{g}$ will be the same in both schemes. The remaining quantities are the interesting ones since they involve one or two traces with one $\gamma_{5}$. In the prescription of [14], one has to define a 'reading point' for these, at which the trace is to be started. Let us discuss our choice for the case of the $C_{F} T_{f}$ part of $\Delta P_{q g}^{(1)}$, in which the incoming particle is a gluon and the outgoing one a quark with the projector $-\gamma_{5} \not h / 4 n \cdot k$ being acted upon. Only topologies (cd),(e),(fg) and (hi) contribute; for the sake of clarity we show the graphs explicitly in Fig. 2. It seems reasonable to choose the projector as the reading point, marked by the

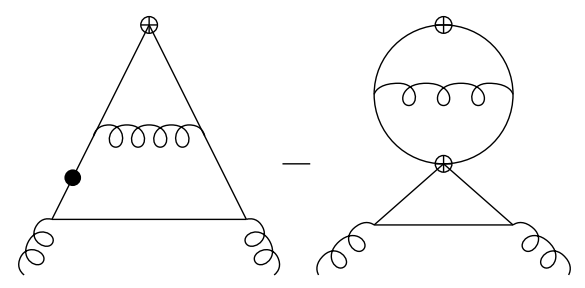

(h)-(i)

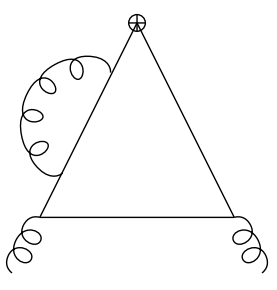

(e)

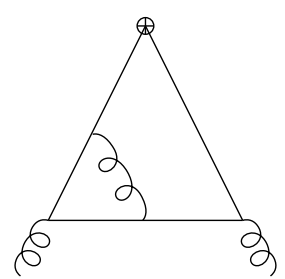

(cd)

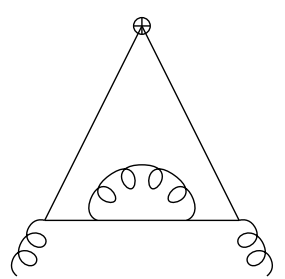

$(f g)$

Figure 2: Graphs for the $C_{F} T_{f}$ part of $\Delta P_{q g}^{(1)}$

'crosses' $\oplus$ in Fig. 2. Doing so for graphs (cd),(e),(fg), which in the language of Eq. (20) represent $\Delta \mathcal{P} K_{0}$, one obtains for each graph exactly the same answer as when using the HVBM $\gamma_{5}$. However, for topology (hi) one has to take care: For the subtraction graph (i), which represents $-\Delta \mathcal{P}\left(K_{0} \Delta \mathcal{P} K_{0}\right)$, the projection operator $\Delta \mathcal{P}$ has by definition effectively cut the Dirac-trace of $(\mathrm{h})\left(=\Delta \mathcal{P}\left(K_{0}^{2}\right)\right)$ into two traces, hereby inserting an additional $\gamma_{5}$ in each trace according to $\Delta L_{q}, \Delta U_{q}$ in (9). Thus (i) essentially becomes the convolution of $\Delta P_{q q}^{(0)}$ for the upper part of the diagram with $\Delta P_{q g}^{(0)}$ for the lower. Since the trace for the upper part then contains two $\gamma_{5}$ which eliminate each other, the $\gamma_{5}$-problem has been shifted from the upper 'cross' to the lower one. This suggests that the trace for graph (h) 
should be read from the black dot rather than from the 'cross'. Indeed, when doing this, it turns out that both pieces (h),(i) yield differences with respect to the HVBM results, which on aggregate are exactly the same as the entries in the column " $\gamma_{5}$ " in Table 2 . This means that we end up with the result in (46) for the $C_{F} N_{f}$ part of $\Delta P_{q g}^{(1)}$, without any extra terms. The same thing happens for the case of the $C_{F}^{2}$ part of $\Delta P_{g q}^{(1)}$, where it is resonable to choose the $-\not p \gamma_{5}$ of the incoming polarized quark as the reading point for the graphs for $\Delta \mathcal{P} K_{0}$, in which case each of these graphs individually gives the same result as in the HVBM scheme. This time, the answer for subtraction graph (i) is also the same as in the HVBM scheme, but when the reading point for graph (h) $\left(\Delta \mathcal{P}\left(K_{0}^{2}\right)\right)$ is again chosen as the quark propagator on the side of the ladder between the rungs, it alone generates all differences in column " $\gamma_{5}$ " of Table 3 , such that the $C_{F}^{2}$ term in (47) is reproduced. Next, we have to calculate the $N_{C} T_{f}$ part of $\Delta P_{q g}^{(1)}$ and the $C_{F} N_{C}$ part of $\Delta P_{g q}^{(1)}$, choosing again $-\gamma_{5} \not / 4 n \cdot k$ and $-\not p \gamma_{5}$ as the reading points, respectively. Here, no extra care has to be taken since for both the sides of the ladder between the rungs in graph (h) are gluons. For each graph the result exactly agrees with the corresponding one in the HVBM scheme. Finally, we have calculated $\Delta P_{q q}^{S,(1)}$, again choosing the projectors as the reading points for its two traces and reproducing once again the result of the calculation in the HVBM scheme. Also, $\Delta C_{q}$ in (49) was obtained in [28] with the photon vertex as reading point.

Thus, to summarize our results for the calculation in the $\gamma_{5}$-scheme of [14], we have indeed reproduced all polarized NLO quantities as given in their final form in Eqs. (44,50), without any extra terms like in (33-39). This is a nice confirmation of our HVBM results and underlines the consistency of the whole calculation. However, it needs to be emphasized that although our choice of the reading points above seems the most reasonable one, other choices should be allowed. But if one insists on choosing the reading point $-\gamma_{5} \not h / 4 n \cdot k$ also for graph (h) of the $C_{F} T_{f}$ part of $\Delta P_{q g}^{(1)}$ (rather than shifting it to the black dot in Fig. 2), the result for this graph is no longer different from the one in the HVBM scheme! Thus a genuinely different final answer for $\Delta P_{q g}^{(1)}$ would be the consequence. We also found that choosing a different reading point in the calculation of $\Delta C_{q}$ the result seems to change. It appears likely that these problems indicate a certain incompatibility of the $\gamma_{5}$-scheme of [14] with the projection method we are using 9 in which, as discussed above, traces sometimes are cut, with insertions of $\gamma_{5}$, by action of $\Delta \mathcal{P}$. The genuinely four-dimensional (i.e., nonanticommuting) $\gamma_{5}$ of 13] seems to be more appropriate here since it gives a unique answer without any extra effort apart from the scheme transformation (40,41).

We mention that we have also recalculated some graphs of the $C_{F} T_{f}$ part of $\Delta P_{q g}^{(1)}$, as well as the coefficient function $\Delta C_{q}$, using the prescription of [15, 18], i.e., eliminating $\gamma_{5}$ via Eq. (27) and contracting the Levi-Civita tensors in $d$ dimensions. This scheme is expected to be equivalent to the HVBM scheme 15. In all cases we studied we indeed obtained the same answer as in the HVBM scheme without having to take into account any $(d-4)$-dimensional scalar products which are not present in this scheme since the metric tensors coming from the products of two $\epsilon$-tensors are taken to be $d$-dimensional. This computational advantage is lost, however, because the trace operations become considerably more involved due to the substitution (27). Furthermore, it is not entirely clear how to generalize this scheme in order to deal with traces with two $\gamma_{5}$, i.e., how to establish equivalence to the HVBM prescription in this case.

\footnotetext{
${ }^{9}$ On the other hand, similar problems with the prescription of [14] also seem to have been the source of an error in a previous version of the OPE calculation of [12].
} 


\subsection{Connection with dimensional reduction}

We finally discuss how our results in Eqs. (44 50) can be translated to dimensional reduction. As was explained in 20,21, the UV sectors of QCD in dimensional regularization and dimensional reduction are made to agree by introducing additional counterterms in the latter which include a finite renormalization of the strong charge. Once this is done, all remaining differences between the results for a NLO quantity in dimensional regularization and in dimensional reduction can only be due to the effects of mass singularities. They are fully accounted for 21,22,23] by the differences between the $d$-dimensional LO splitting functions (as to be obtained in dimensional regularization) and the four-dimensional ones (corresponding to dimensional reduction). This makes it very easy to transform our results in Eqs. (44 50) to dimensional reduction: We just need to perform a factorization scheme transformation (40), with the $\Delta z_{i j}$ to be obtained from the parts $\sim \epsilon$ of the polarized $d$-dimensional LO splitting functions as obtained in the HVBM scheme. The latter read:

$$
\begin{aligned}
\Delta P_{q q}^{(0), d=4-2 \epsilon}(x) & =C_{F}\left\{\frac{2}{[1-x]_{+}}-1-x+3 \epsilon(1-x)+\frac{3+\epsilon}{2} \delta(1-x)\right\} \\
\Delta P_{q g}^{(0), d=4-2 \epsilon}(x) & =2 T_{f}\{2 x-1-2 \epsilon(1-x)\} \\
\Delta P_{g q}^{(0), d=4-2 \epsilon}(x) & =C_{F}\{2-x+2 \epsilon(1-x)\} \\
\Delta P_{g g}^{(0), d=4-2 \epsilon}(x) & =2 N_{C}\left\{\frac{1}{[1-x]_{+}}-2 x+1+2 \epsilon(1-x)\right\}+\left(\frac{\beta_{0}}{2}+\frac{N_{C}}{6} \epsilon\right) \delta(1-x)(58
\end{aligned}
$$

However, care has to be taken: We have already performed the scheme transformation (41) which effectively amounted to removing $4 \epsilon(1-x)$ from $\Delta P_{q q}^{(0), d=4-2 \epsilon}$, hereby rendering it equal to its unpolarized counterpart, $P_{q q}^{(0), d=4-2 \epsilon}$ (see Eq. (42)). We thus have to use

$$
\Delta P_{q q}^{(0), d=4-2 \epsilon}(x)=C_{F}\left\{\frac{2}{[1-x]_{+}}-1-x-\epsilon(1-x)+\frac{3+\epsilon}{2} \delta(1-x)\right\}
$$

instead of (55). From the parts $\sim \epsilon$ in Eqs. (56-59) we can now read off the $\Delta z_{i j}$ to be used in the new scheme transformation (40):

$$
\begin{aligned}
& \Delta z_{q q}(x)=\frac{C_{F}}{4}\left((1-x)-\frac{1}{2} \delta(1-x)\right), \quad \Delta z_{q g}(x)=T_{f}(1-x), \\
& \Delta z_{g q}(x)=-\frac{C_{F}}{2}(1-x), \quad \Delta z_{g g}(x)=-N_{C}\left((1-x)+\frac{1}{24} \delta(1-x)\right) .
\end{aligned}
$$

Inserting these into (40), one finds:

$$
\begin{aligned}
\Delta P_{q q}^{(1), \mathrm{DR}}-\Delta P_{q q}^{(1)}(x) & =-\frac{\beta_{0} C_{F}}{2}\left((1-x)-\frac{1}{2} \delta(1-x)\right)-4 C_{F} T_{f}(1-x) \ln x, \\
\Delta P_{q g}^{(1), \mathrm{DR}}-\Delta P_{q g}^{(1)}(x) & =C_{F} T_{f}(9-10 x+6 \ln x-8(1-x) \ln (1-x)) \\
& +N_{C} T_{f}\left(-\frac{25}{3}+\frac{26}{3} x+8(1-x)(\ln (1-x)-\ln x)\right) \\
\Delta P_{g q}^{(1), \mathrm{DR}}-\Delta P_{g q}^{(1)}(x) & =C_{F}^{2}\left(5-\frac{9}{2} x-4(1-x) \ln (1-x)+(4-x) \ln x\right)
\end{aligned}
$$




$$
\begin{aligned}
& +C_{F} N_{C}\left(3-\frac{19}{6} x+4(1-x) \ln (1-x)\right)-\frac{8}{3} C_{F} T_{f}(1-x) \\
\Delta P_{g g}^{(1), \mathrm{DR}}-\Delta P_{g g}^{(1)}(x) & =2 \beta_{0} N_{C}\left((1-x)+\frac{1}{24} \delta(1-x)\right)+4 C_{F} T_{f}(1-x) \ln x,
\end{aligned}
$$

where the $\Delta P_{i j}^{(1)}$ are our final results in Eqs. $44-48$ and the $\Delta P_{i j}^{(1), \mathrm{DR}}$ are the polarized NLO $(\overline{\mathrm{MS}})$ splitting functions in dimensional reduction. For the $q q$ sector we have only written down the entry of the singlet matrix, $\Delta P_{q q}^{(1)}=\Delta P_{q q}^{+,(1)}+\Delta P_{q q}^{S,(1)}$ (see Eq. (19)). Furthermore, we have not written down the short-distance cross sections since their transformation is trivial. We mention however, that the effect of $\Delta z_{q g}$ on $\Delta C_{g}$ is to remove the term $+2(1-x)$ at the end of $(50)$, which corresponds to a factorization scheme in which gluons contribute to the first moment ( $x$-integral) of the structure function $g_{1}$. Such a factorization scheme was suggested in [32. Since furthermore the first moments of $\Delta P_{q q}^{(1), \mathrm{DR}}$ and $\Delta P_{q g}^{(1), \mathrm{DR}}$ vanish, it follows that the total polarization of quarks and antiquarks, $\int_{0}^{1} d x \Delta \Sigma^{\mathrm{DR}}\left(x, Q^{2}\right)$, is $Q^{2}$-independent when defined in dimensional reduction.

As a little cross-check on our findings in Eqs. (61-64) we have recalculated all polarized NLO splitting functions, using all matrix elements in four dimensions (i.e., setting $\epsilon$ and ( $d-$ 4)-dimensional scalar products to zero), but performing loop and phase space integrations in $d$ dimensions after all spin algebra has been done. Subtracting the corresponding results from our HVBM ones, Eqs. (44-48), we reproduced all logarithmic terms in Eqs. (61-64), leaving only some non-logarithmic pieces (and of course the $\delta$-functions) unaccounted for. It is precisely such non-logarithmic terms one expects to be generated by inclusion of the additional finite UV-counterterms needed for a full and proper calculation in dimensional reduction 20,21].

The main reason for performing our exercise concerning dimensional reduction is that the results for $\Delta P_{i j}^{(1), \mathrm{DR}}$ to be obtained from Eqs. 61-64, (44, 48) fulfil a remarkable relation if one sets $C_{F}=N_{C}=2 T_{f} \equiv N$ (see also 12]):

$$
\Delta P_{q q}^{(1), \mathrm{DR}}(x)+\Delta P_{g q}^{(1), \mathrm{DR}}(x)-\Delta P_{q g}^{(1), \mathrm{DR}}(x)-\Delta P_{g g}^{(1), \mathrm{DR}}(x) \equiv 0
$$

which is known to hold for LO splitting functions and also for the unpolarized NLO splitting functions in dimensional reduction [33,22], and is expected from supersymmetry.

\section{Summary}

We have presented a detailed description of our calculation [11] of the spin-dependent NLO GLAP splitting functions in the method of [6, 国5]. The main calculation has been performed using the $\gamma_{5}$-prescription of [13], but we have also discussed the results one obtains when using a fully anticommuting $\gamma_{5}$, giving up cyclicity of the Dirac-trace 114. The results for the two schemes turn out to be the same for a reasonably chosen reading point in the latter scheme, but the HVBM scheme appears to be the safer and the more straightforward prescription. Our final result confirms the previous OPE results of [12]. We have also provided the connection with dimensional reduction, in which the NLO splitting functions satisfy a simple supersymmetric relation. 


\section{Acknowledgements}

I am thankful to R.K. Ellis and M. Stratmann for helpful discussions. 


\begin{tabular}{|c|c|c|c|c|c|c|c|c|c|c|c|c|c|c|}
\hline$\Delta P_{q q}^{V,(1)}(x)-P_{q q}^{V,(1)}(x)$ & \multicolumn{5}{|c|}{$C_{F}^{2}$} & \multicolumn{6}{|c|}{$C_{F} N_{C}$} & \multicolumn{3}{|c|}{$C_{F} T_{f}$} \\
\hline $\begin{array}{l}\text { Terms originating from } \\
(d-4) \text {-dimensional scalar products }\end{array}$ & (b) & $(\mathrm{cd})$ & (e) & (hi) & Sum & (b) & $(c d)$ & $(\mathrm{fg})$ & Sum & $" \gamma_{5} "$ & final & $(\mathrm{fg}), \mathrm{Sum}$ & $" \gamma_{5} "$ & final \\
\hline $\begin{array}{l}(1-x) \ln (x) \\
(1-x) \ln (1-x) \\
(1-x) I_{0} \\
1-x\end{array}$ & & $\begin{array}{l}-4 \\
-8 \\
6\end{array}$ & $\begin{array}{c}8 \\
-6\end{array}$ & -4 & & & $\begin{array}{l}-4 \\
-4\end{array}$ & $\begin{array}{r}4 \\
4 \\
-4\end{array}$ & -4 & $11 / 3$ & $-1 / 3$ & 2 & $-4 / 3$ & $2 / 3$ \\
\hline \multicolumn{15}{|l|}{ Other terms } \\
\hline $\begin{array}{l}(1-x) \ln (x) \\
(1-x) \ln (1-x) \\
(1-x) I_{0} \\
1-x\end{array}$ & & $\begin{array}{l}-4 \\
-8 \\
6\end{array}$ & $\begin{array}{l}8 \\
8 \\
-6\end{array}$ & -4 & & & $\begin{array}{l}-4 \\
-4\end{array}$ & $\begin{array}{c}4 \\
4 \\
-10 / 3\end{array}$ & $-10 / 3$ & $11 / 3$ & $1 / 3$ & $2 / 3$ & $-4 / 3$ & $-2 / 3$ \\
\hline
\end{tabular}

Table 1: qq-Diagrams: Results for the difference $\Delta P_{q q}^{V,(1)}(x)-P_{q q}^{V,(1)}(x)$ 


\begin{tabular}{|c|c|c|c|c|c|c|c|c|c|c|c|}
\hline$\Delta P_{q g}^{(1)}(x)$ & \multicolumn{7}{|c|}{$C_{F} T_{f}$} & \multicolumn{4}{|c|}{$N_{C} T_{f}$} \\
\hline Terms & (cd) & (e) & $(f g)$ & (hi) & Sum & $" \gamma_{5} "$ & final & (b) & $(\mathrm{cd})$ & (hi) & Sum \\
\hline$\delta p_{q g}(x) \ln ^{2}(1-x)$ & 2 & & -2 & 2 & 2 & & 2 & & & -2 & -2 \\
\hline$\delta p_{q g}(x) \ln ^{2}(x)$ & 2 & -4 & & 3 & 1 & & 1 & -1 & & -2 & -3 \\
\hline$\delta p_{q g}(x) \ln (x) \ln (1-x)$ & 4 & -8 & -4 & 4 & -4 & & -4 & & 4 & -4 & \\
\hline$\delta p_{q g}(x) I_{0}(\ln (1-x)+\ln (x))$ & 8 & -8 & -4 & 4 & & & & & 4 & -4 & \\
\hline$\delta p_{q g}(x) \ln (x)$ & -4 & 8 & 3 & 1 & 8 & -8 & & 5 & -1 & 4 & 8 \\
\hline$\delta p_{q g}(x) \ln (1-x)$ & -2 & 6 & & -8 & -4 & & -4 & 4 & & & 4 \\
\hline$\delta p_{q g}(x) \mathrm{Li}_{2}(1-x)$ & -8 & & & 8 & & & & & 8 & -8 & \\
\hline$\delta p_{q g}(x) \pi^{2} / 3$ & 6 & -4 & -2 & -2 & -2 & & -2 & & -1 & 2 & 1 \\
\hline$\delta p_{q g}(-x) S_{2}$ & & & & & & & & -2 & & & -2 \\
\hline$\delta p_{q g}(x) I_{0}$ & & 8 & & -8 & & & & 4 & -4 & & \\
\hline$\delta p_{q g}(x) I_{1}$ & -8 & 8 & 4 & -4 & & & & & -4 & 4 & \\
\hline$\delta p_{q g}(x)$ & -20 & 14 & 11 & & 5 & & 5 & & 2 & & 2 \\
\hline $\ln ^{2}(x)$ & & & & & & & & & & -6 & -6 \\
\hline $\ln (x)$ & 6 & -8 & & 9 & 7 & -16 & -9 & 11 & 3 & -4 & 10 \\
\hline $\ln (1-x)$ & 4 & & -4 & 4 & 4 & & 4 & & & -4 & -4 \\
\hline$I_{0}$ & 8 & -8 & -4 & 4 & & & & & 4 & -4 & \\
\hline $1-x$ & -10 & 12 & 7 & -2 & 7 & -24 & -17 & 22 & 4 & & 26 \\
\hline
\end{tabular}

Table 2: Results for the $q g$ diagrams 


\begin{tabular}{|c|c|c|c|c|c|c|c|c|c|c|c|c|}
\hline$\Delta P_{g q}^{(1)}(x)$ & \multicolumn{6}{|c|}{$C_{F}^{2}$} & \multicolumn{5}{|c|}{$C_{F} N_{C}$} & $C_{F} T_{f}$ \\
\hline Terms & $(\mathrm{cd})$ & $(\mathrm{fg})$ & (hi) & Sum & $" \gamma_{5} "$ & final & (b) & $(\mathrm{cd})$ & (e) & (hi) & Sum & (e),Sum \\
\hline$\delta p_{g q}(x) \ln ^{2}(1-x)$ & 1 & -1 & -1 & -1 & & -1 & & & & 1 & 1 & \\
\hline$\delta p_{g q}(x) \ln ^{2}(x)$ & & & $1 / 2$ & $1 / 2$ & & $1 / 2$ & $-1 / 2$ & 1 & -2 & & $-3 / 2$ & \\
\hline$\delta p_{g q}(x) \ln (x) \ln (1-x)$ & 4 & -2 & -2 & & & & & & -4 & 2 & -2 & \\
\hline$\delta p_{g q}(x) I_{0}(\ln (1-x)+\ln (x))$ & 4 & -2 & -2 & & & & & 2 & -4 & 2 & & \\
\hline$\delta p_{g q}(x) \ln (x)$ & -2 & $3 / 2$ & -4 & $-9 / 2$ & 4 & $-1 / 2$ & 3 & -2 & 8 & 4 & 13 & \\
\hline$\delta p_{g q}(x) \ln (1-x)$ & -3 & 2 & 2 & 1 & & 1 & 2 & & $11 / 3$ & -6 & $-1 / 3$ & $-4 / 3$ \\
\hline$\delta p_{g q}(x) \operatorname{Li}_{2}(1-x)$ & 4 & & -4 & & & & & -4 & & 4 & & \\
\hline$\delta p_{g q}(x) \pi^{2} / 3$ & & -1 & 1 & & & & & $5 / 2$ & -2 & -1 & $-1 / 2$ & \\
\hline$\delta p_{g q}(-x) S_{2}$ & & & & & & & -1 & & & & -1 & \\
\hline$\delta p_{g q}(x) I_{0}$ & -4 & 2 & 2 & & & & 2 & -4 & 8 & -6 & & \\
\hline$\delta p_{g q}(x) I_{1}$ & -4 & 2 & 2 & & & & & -2 & 4 & -2 & & \\
\hline$\delta p_{g q}(x)$ & -10 & $11 / 2$ & & $-9 / 2$ & & $-9 / 2$ & & 1 & $67 / 9$ & & $76 / 9$ & $-20 / 9$ \\
\hline $\ln ^{2}(x)$ & & & & & & & & & & 6 & 6 & \\
\hline $\ln (x)$ & -2 & & 17 & 15 & -16 & -1 & -11 & 1 & -8 & -4 & -22 & \\
\hline $\ln (1-x)$ & 4 & -4 & -4 & -4 & & -4 & & & & 4 & 4 & \\
\hline$I_{0}$ & 8 & -4 & -4 & & & & & 4 & -8 & 4 & & \\
\hline $1-x$ & 5 & $-7 / 2$ & 11 & $25 / 2$ & -12 & $1 / 2$ & -11 & -2 & $-22 / 3$ & 8 & $-37 / 3$ & $8 / 3$ \\
\hline
\end{tabular}

Table 3: Results for the $g q$ diagrams 


\begin{tabular}{|c|c|c|c|c|c|c|c|c|c|c|c|c|c|c|c|}
\hline$\Delta P_{g g}^{(1)}(x)$ & \multicolumn{3}{|c|}{$C_{F} T_{f}$} & \multicolumn{5}{|c|}{$N_{C} T_{f}$} & \multicolumn{7}{|c|}{$N_{C}^{2}$} \\
\hline Terms & (b) & (hi) & Sum & (b) & $(c d)$ & $(\mathrm{e})$ & $(\mathrm{fg})$ & Sum & (b) & $(\mathrm{cd})$ & $(\mathrm{e})$ & $(\mathrm{fg})$ & (hi) & $(\mathrm{jk})$ & Sum \\
\hline$\delta p_{g g}(x) \ln ^{2}(1-x)$ & & & & & & & & & & 2 & & -2 & & & \\
\hline$\delta p_{g g}(x) \ln ^{2}(x)$ & & & & & & & & & -1 & 2 & -4 & & 4 & & 1 \\
\hline$\delta p_{g g}(x) \ln (x) \ln (1-x)$ & & & & & & & & & & 8 & -8 & -4 & & & -4 \\
\hline$\delta p_{g g}(x) I_{0}(\ln (1-x)+\ln (x))$ & & & & & & & & & & 12 & -8 & -4 & & & \\
\hline$\delta p_{g g}(x) \ln (x)$ & & & & & $4 / 3$ & & $-4 / 3$ & & & $-11 / 3$ & & $11 / 3$ & & & \\
\hline$\delta p_{g g}(x) \ln (1-x)$ & & & & & $8 / 3$ & $-8 / 3$ & & & 4 & $2 / 3$ & $22 / 3$ & -4 & -8 & & \\
\hline$\delta p_{g g}(x) \pi^{2} / 3$ & & & & & & & & & & 5 & -4 & -2 & & & -1 \\
\hline$\delta p_{g g}(-x) S_{2}$ & & & & & & & & & -2 & & & & & & -2 \\
\hline$\delta p_{g g}(x) I_{0}$ & & & & & & & & & 4 & 8 & & -4 & -8 & & \\
\hline$\delta p_{g g}(x) I_{1}$ & & & & & & & & & & -12 & 8 & 4 & & & \\
\hline$\delta p_{g g}(x)$ & & & & & $40 / 9$ & $-40 / 9$ & $-20 / 9$ & $-20 / 9$ & & $-170 / 9$ & $134 / 9$ & $103 / 9$ & & & $67 / 9$ \\
\hline$(1+x) \ln ^{2}(x)$ & & -2 & -2 & & & & & & & & & & 4 & & 4 \\
\hline$(1+x) \ln (x)$ & 8 & -12 & -4 & -4 & $8 / 3$ & & & $-4 / 3$ & $-21 / 2$ & $-41 / 6$ & & & 8 & 3 & $-19 / 3$ \\
\hline$(1-x) \ln (x)$ & & -6 & -6 & & & & & & & -8 & 16 & & 8 & & 16 \\
\hline$(1-x) \ln (1-x)$ & & & & & & & & & & -8 & & 8 & & & \\
\hline$(1-x) I_{0}$ & & & & & & & & & & -24 & 16 & 8 & & & \\
\hline $1-x$ & 16 & -26 & -10 & -8 & $-5 / 3$ & $16 / 3$ & $1 / 3$ & -4 & $-233 / 8$ & $7 / 3$ & $-44 / 3$ & $-95 / 12$ & 32 & $63 / 8$ & $-19 / 2$ \\
\hline
\end{tabular}

Table 4: Results for the $g g$ diagrams 


\section{Appendix}

Almost all technical ingredients we need, like two- and three-point functions and threeparticle phase space integrals, have recently been given in the documentation [8] of the unpolarized calculation. We only need to consider some new details related to the treatment of $\gamma_{5}$ and $\epsilon^{\mu \nu \rho \sigma}$ in $d$ dimensions, which can introduce explicit dependence of the matrix element squared on scalar products of the nonphysical ( $d$-4-dimensional) components of vectors (see section 2.4). It is most convenient to work in the IMF parametrization of the momenta [4]:

$$
\begin{aligned}
p & =\left(P, \overrightarrow{0}_{x y}, P, \overrightarrow{0}_{d-4}\right) \\
n & =\left(\frac{p n}{2 P}, \overrightarrow{0}_{x y},-\frac{p n}{2 P}, \overrightarrow{0}_{d-4}\right) \\
k & =\left(x P+\frac{k^{2}+\tilde{k}^{2}}{4 x P}, \vec{k}_{T}, x P-\frac{k^{2}+\tilde{k}^{2}}{4 x P}, \hat{\vec{k}}\right)
\end{aligned}
$$

where $p$ represents the incoming and $k$ the outgoing ('observed') parton, with

$$
\tilde{k}^{2} \equiv k_{x}^{2}+k_{y}^{2}+(\hat{\vec{k}})^{2} \equiv k_{T}^{2}+\hat{k}^{2}
$$

being the total transverse momentum squared of $k$ relative to the longitudinal axis defined by $p, n$. Here we have explicitly introduced the $(d-4)$-dimensional components of $k$ with

$$
\hat{k}^{2} \equiv(\hat{\vec{k}})^{2}=-\hat{g}_{\mu \nu} k^{\mu} k^{\nu},
$$

$\hat{g}_{\mu \nu}$ being the $(d-4)$-dimensional metric tensor of section 2.4. The labelling of the other momenta is fixed in Fig. 3.

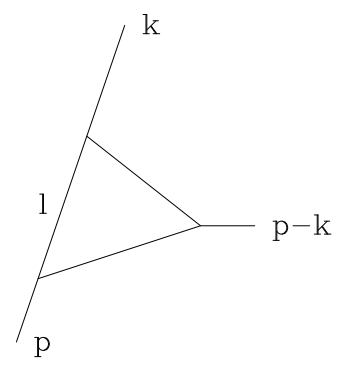

(a)

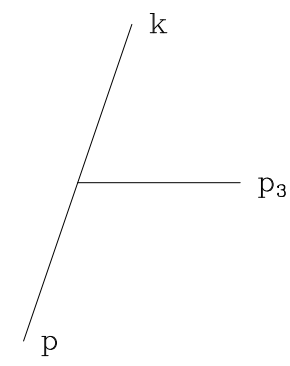

(b)

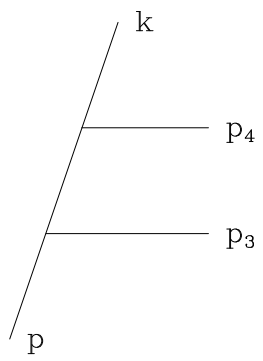

(c)

Figure 3: (a) Vertex correction graph (b) One parton emission (c) Two parton emission.

\section{A Virtual Integrals}

The virtual diagrams always comprise emission of only one real massless particle (the rung of the ladder) from the process $p \rightarrow k+p_{3}$ (see Fig. 3(a),(b)). The corresponding phase 
space reads (including the integration over the 'observed' parton $k$ ):

$$
\begin{aligned}
P S^{(1)} & =\int d^{d} k x \delta\left(x-\frac{n \cdot k}{p n}\right) \int \frac{d^{d} p_{3}}{(2 \pi)^{d-1}} \delta^{+}\left(p_{3}^{2}\right)(2 \pi)^{d} \delta^{d}\left(p-p_{3}-k\right) \\
& =\frac{x \pi^{2-\epsilon}}{\Gamma(1-\epsilon)}(1-x)^{-\epsilon} \int_{0}^{Q^{2}} d\left|k^{2}\right|\left|k^{2}\right|^{-\epsilon}\left((-\epsilon) \int_{0}^{1} d \hat{\kappa} \hat{\kappa}^{-1-\epsilon}\right)
\end{aligned}
$$

where $\hat{\kappa}$ is defined by

$$
\hat{k}^{2}=\left|k^{2}\right|(1-x) \hat{\kappa} .
$$

The last integral in Eq. (A.4) has been written in such a way that it is unity if there is no dependence on $\hat{\kappa}$, i.e., $\hat{k}^{2}$. As we can also see, any term proportional $\hat{k}^{2}$ in the matrix element will give a factor $\epsilon$ after performing the $\hat{\kappa}$-integration.

When performing a loop calculation in the polarized case, the matrix element will in general not only depend on $\hat{k}^{2}$, but also on scalar products involving the $(d-4)$-dimensional components of the loop momentum $l$, like $\hat{l}^{2}, \hat{\vec{k}} \cdot \hat{\vec{l}}$. One can always write, e.g., $\hat{\vec{l}} \cdot \hat{\vec{k}}$ as $-\hat{g}_{\mu \nu} l^{\mu} k^{\nu}$, then perform the loop integration, and contract the result with $-\hat{g}_{\mu \nu} k^{\nu}$ afterwards. Obviously, this requires knowledge of vectorial two- or three-point functions (and tensorial ones when $\hat{l}^{2}=-\hat{g}_{\mu \nu} l^{\mu} l^{\nu}$ appears). These can be easily obtained from the scalar ones presented in [8]. The only new feature is that, unlike in the unpolarized calculation, we also need the vectorial three-point functions with a light-cone gauge propagator,

$$
J_{3, n}^{\mu} \equiv \int \frac{d^{d} l}{(2 \pi)^{d}} \mathrm{PV}\left(\frac{p_{+}}{l_{+}}\right) \frac{l^{\mu}}{\left(l^{2}+i \varepsilon\right)\left((l-k)^{2}+i \varepsilon\right)\left((l-p)^{2}+i \varepsilon\right)},
$$

where $p_{+}=p n, l_{+}=n \cdot l$, and 'PV' denotes use of the principal value prescription (22) in order to regularize the singularities of the light-cone gauge propagator. More precisely, since $J_{3, n}^{\mu}$ will be contracted with $-\hat{g}_{\mu \nu} k^{\nu}$ afterwards, we only need its part $\sim k^{\mu}$, as $p, n$ do not possess non-vanishing components in their $(d-4)$-dimensional parts. Assuming Lorentz covariance of the integral one finds

$$
J_{3, n}^{\mu}=\frac{1}{2(1-x)}\left(J_{3, n}-J_{3}+\frac{1}{\left|k^{2}\right|} J_{2, n}\right) k^{\mu}+\ldots,
$$

where $J_{2, n}, J_{3, n}$ are the scalar two- and three-point functions with a light-cone gauge propagator as given in Eqs. (A.4) and (A.14) of [8], respectively, and $J_{3}$ is the ordinary scalar three-point function of (A.13) in [8]. The dots in (A.7) indicate the contributions proportional to $p^{\mu}$ and $n^{\mu}$, which we do not need. We finally note that in our previous paper [1]] on the polarized NLO splitting functions we were able to determine the results for most of the virtual graphs just from their contributions in the unpolarized case. The results in Appendix A of [8] and the ones presented here now enable a straightforward direct calculation of all virtual contributions also for the polarized case.

\section{B Real Integrals}

The real emission processes $p \rightarrow k+p_{3}+p_{4}$ (see Fig. 3(c)) comprise integration over the momenta of two unobserved real massless particles. In addition to A.1 we define

$$
p_{3}=\left(p_{3}^{0}, \vec{p}_{3}^{x y}, p_{3}^{z}, \hat{\vec{p}}_{3}\right),
$$


introducing the $(d-4)$-dimensional components of $p_{3}$ which we split into a part $\hat{p}_{3}^{\|}$parallel to those of $k$ and a transverse part $\hat{p}_{3}^{\perp}$. The phase space then reads (again including the integration over the 'observed' parton $k$ ):

$$
\begin{aligned}
P S^{(2)}= & \int d^{d} k x \delta\left(x-\frac{n \cdot k}{p n}\right) \int \frac{d^{d} p_{3}}{(2 \pi)^{d-1}} \delta^{+}\left(p_{3}^{2}\right) \int \frac{d^{d} p_{4}}{(2 \pi)^{d-1}} \delta^{+}\left(p_{4}^{2}\right)(2 \pi)^{d} \delta^{d}\left(p-k-p_{3}-p_{4}\right) \\
= & \frac{1}{2^{4+2 \epsilon} \pi \Gamma(1-2 \epsilon)} x^{\epsilon}(1-x)^{1-2 \epsilon} \int_{0}^{Q^{2}} d\left|k^{2} \| k^{2}\right|^{1-2 \epsilon} \\
& \times \int_{0}^{1} d \tilde{\kappa}(\tilde{\kappa}(1-\tilde{\kappa}))^{-\epsilon} \int_{0}^{1} d w(w(1-w))^{-\epsilon} \int_{0}^{1} d v(v(1-v))^{-\frac{1}{2}-\epsilon} \\
& \times\left((-\epsilon) \int_{0}^{1} d \hat{\kappa} \hat{\kappa}^{-1-\epsilon}\right)\left(\left(-\frac{1}{2}-\epsilon\right) \int_{0}^{1} d \lambda^{\perp}\left(\lambda^{\perp}\right)^{-3 / 2-\epsilon}\right)\left(\frac{1}{\pi} \int_{0}^{1} d \lambda^{\|}\left(\lambda^{\|}\left(1-\lambda^{\|}\right)\right)^{-1 / 2}\right)
\end{aligned}
$$

where we have defined

$$
\begin{aligned}
\hat{k}^{2} & =\left|k^{2}\right|(1-x) \hat{\kappa} \tilde{\kappa}, \\
\tilde{k}^{2} & =\left|k^{2}\right|(1-x) \tilde{\kappa}, \\
p_{3}^{0}+p_{3}^{z} & =2 P(1-x) w=2 P \frac{n \cdot p_{3}}{p n}, \\
\left(p_{3}^{0}\right)^{2}-\left(p_{3}^{z}\right)^{2} & =c_{1}^{2}+v\left(c_{2}^{2}-c_{1}^{2}\right)=\frac{1}{P}\left(p_{3}^{0}+p_{3}^{z}\right)\left(p \cdot p_{3}\right), \\
\hat{p}_{3}^{\|} & =\lambda_{1}+\lambda^{\|}\left(\lambda_{2}-\lambda_{1}\right), \\
\left(\hat{p}_{3}^{\perp}\right)^{2} & =v(1-v)\left(c_{1}+c_{2}\right)^{2} \lambda^{\perp}
\end{aligned}
$$

with

$$
\begin{aligned}
c_{1,2} & \equiv \sqrt{\frac{\left|k^{2}\right|(1-x) w}{x}}[\sqrt{(1-w)(1-\tilde{\kappa})} \mp \sqrt{x w \tilde{\kappa}}], \\
\lambda_{1,2} & =-\frac{1}{2} \frac{\hat{\kappa}}{\hat{k} w}\left(\left(p_{3}^{0}\right)^{2}-\left(p_{3}^{z}\right)^{2}-c_{1} c_{2}\right) \mp\left(c_{1}+c_{2}\right) \sqrt{(1-\hat{\kappa})\left(1-\lambda^{\perp}\right) v(1-v)}
\end{aligned}
$$

Again the last three integrals in Eq. (B.9) have been written in such a way that they are all unity if there is no dependence on $(d-4)$-dimensional scalar products. If present, such terms only give contributions proportional to $\epsilon$ after the last three integrals have been performed. The advantage of writing $P S^{(2)}$ in this way and performing the three last integrals first is that the result of these integrations can always be expressed in terms of usual ( $d$-dimensional) scalar products. For instance,

$$
\begin{aligned}
1 & \longrightarrow 1 \\
\hat{k}^{2} & \longrightarrow-\frac{\epsilon}{1-\epsilon}\left(\left|k^{2}\right|+2 x(p \cdot k)\right), \\
\hat{p}_{3}^{2} \equiv\left(\hat{p}_{3}^{\|}\right)^{2}+\left(\hat{p}_{3}^{\perp}\right)^{2} & \longrightarrow-\frac{2 \epsilon}{1-\epsilon}\left(p \cdot p_{3}\right) \frac{n \cdot p_{3}}{p n}, \\
-\hat{g}_{\mu \nu} k^{\mu} p_{3}^{\nu} \equiv \hat{k} \hat{p}_{3}^{\|} & \longrightarrow-\frac{\epsilon}{1-\epsilon}\left(-(1-x)\left(p \cdot p_{3}\right)-\frac{\left|k^{2}\right|}{2}-(p \cdot k)+(p \cdot k) \frac{n \cdot p_{3}}{p n}\right)
\end{aligned}
$$


after integration over $\hat{\kappa}, \lambda^{\perp}$ and $\lambda^{\|}$. The other two $(d-4)$-dimensional scalar products that appear in the calculation, $\hat{k}^{2} \hat{p}_{3}^{2}$ and $\left(\hat{k} \hat{p}_{3}^{\|}\right)^{2}$, have lengthy expressions which can be straightforwardly obtained from (B.9). Thus, after performing the last three integrations in (B.9), there are only terms left in the matrix element which are familiar from the unpolarized case. The matrix element can then be further integrated using the results of Appendix B of [8] or, equivalently, doing the remaining integrals in Eq. (B.9).

\section{References}

[1] G. Altarelli and G. Parisi, Nucl. Phys. B126 (1977) 298.

[2] Yu. L. Dokshitzer Sov. Phys. JETP 46 (1977) 641;

cf. L. N. Lipatov, Sov. J. Nucl. Phys. 20 (1975) 95;

V.N. Gribov and L.N. Lipatov, Sov. J. Nucl. Phys. 15 (1972) 438.

[3] E.G. Floratos, D.A. Ross, and C.T. Sachrajda, Nucl. Phys. B129 (1977) 66; E: B139 (1978) 545; Nucl. Phys. B152 (1979) 493;

see also: A. Gonzales-Arroyo, C. Lopez, and F.J. Yndurain, Nucl. Phys. B153 (1979) 161; A. Gonzales-Arroyo and C. Lopez, Nucl. Phys. B166 (1980) 429; E.G. Floratos, C. Kounnas, and R. Lacaze, Phys. Lett. 98B (1981) 89,285; Nucl. Phys. B192 (1981) 417.

[4] G. Curci, W. Furmanski and R. Petronzio, Nucl. Phys. B175 (1980) 27.

[5] W. Furmanski and R. Petronzio, Phys. Lett. 97B (1980) 437.

[6] R.K. Ellis, H. Georgi, M. Machacek, H.D. Politzer, and G.G. Ross, Phys. Lett. 78B (1978) 281; Nucl. Phys. B152 (1979) 285.

[7] R. Hamberg, PhD Thesis, University of Leiden, 1991;

R. Hamberg and W. van Neerven, Nucl. Phys. B379 (1992) 143.

[8] R.K. Ellis and W. Vogelsang, CERN-TH/96-50, RAL-TR-96-012, hep-ph/9602356.

[9] G. Leibbrandt, Phys. Rev. D29 (1984) 1699;

For an overview see also: G. Leibbrandt, Rev. Mod. Phys. 59 (1987) 1067.

[10] A. Andrasi and J. C. Taylor, Nucl. Phys. B310 (1988) 222.

[11] W. Vogelsang, Rutherford report, RAL-TR-95-071, hep-ph/9512218.

[12] R. Mertig and W.L. van Neerven, Univ. Leiden INLO-PUB-6/95 and NIKHEF-H/95031, June 1995, November 1995 (revised).

[13] G. 't Hooft and M. Veltman, Nucl. Phys. B44 (1972) 189; P. Breitenlohner and D. Maison, Comm. Math. Phys. 52 (1977) 11.

[14] J.G. Körner, D. Kreimer, and K. Schilcher, Zeit. Phys. C54 (1992) 503.

[15] S.A. Larin and J.A.M. Vermaseren, Phys. Lett. B259 (1991) 345; S.A. Larin, Phys. Lett. B303 (1993) 113. 
[16] M. Chanowitz, M. Furman, and I. Hinchliffe, Nucl. Phys. B159 (1979) 225.

[17] M. Jamin and M.E. Lautenbacher, TU München report TUM-T31-20/91.

[18] E.B. Zijlstra and W.L. van Neerven, Phys. Lett. B297 (1992) 377; Nucl. Phys. B417 (1994) 61; E: B426 (1994) 245.

[19] W. Siegel, Phys. Lett. 84B (1979) 193.

[20] G.A. Schuler, S. Sakakibara, and J.G. Körner, Phys. Lett. B194 (1987) 125.

[21] J.G. Körner and M.M. Tung, Zeit. Phys. C64 (1994) 255.

[22] Z. Kunszt, A. Signer, and Z. Trocsanyi, Nucl. Phys. B411 (1994) 397.

[23] B. Kamal, Phys. Rev. D53 (1996) 1142.

[24] M.A. Ahmed and G.G. Ross, Nucl. Phys. B111 (1976) 441.

[25] R.T. Herrod and S. Wada, Phys. Lett. 96B (1980) 195.

[26] A. Devoto and D.W. Duke, Nuov. Cim. (Riv.) 7 (1984) 1.

[27] M. Glück, E. Reya, M. Stratmann, and W. Vogelsang, Univ. Dortmund DO-TH 95/13, Rutherford RAL-TR-95-042, to appear in Phys. Rev. D.

[28] M. Stratmann, W. Vogelsang, and A. Weber, Phys. Rev. D53 (1996) 138.

[29] J. Kodaira, S. Matsuda, K. Sasaki, and T. Uematsu, Nucl. Phys. B159 (1979) 99.

[30] M. Glück and E. Reya, Phys. Rev. D25 (1982) 1211.

[31] A. Weber, Nucl. Phys. B382 (1992) 63.

[32] G. Altarelli and G.G. Ross, Phys. Lett. B212 (1988) 391; G. Altarelli and W.J. Stirling, Particle World 1 (1989) 40; G. Altarelli and B. Lampe, Zeit. Phys. C47 (1990) 315.

[33] I. Antoniadis and E.G. Floratos, Nucl. Phys. B191 (1981) 217. 